\title{
Towards an understanding of -ward(s) prepositions: a sociohistorical exploration of the distribution of near-identical variants
}

by Mary Bisbee

\section{Introduction}

English has a peculiar class of prepositions that exist in two nearly identical forms, one form with a zero ending $(-\varnothing)$ and the other with an s (-s, phonetically often $/ z /$ ), all of them containing the Old English suffix -ward. Examples include toward(s), forward(s), onward(s), inward(s), and homeward(s). As language is, by its nature, systematic, it seems unlikely that the occurrence of one form instead of another is totally arbitrary. This project aims to explore some of the conditioning factors in the preference of one form over another in written as well as colloquial language.

First of all, I would like to defend my position of referring to these -ward words as "prepositions" when they seem to have many properties of adverbs, including often modifying a verb and identifying a manner, as in the sentence "He walked backward(s)." However, The Cambridge grammar of the English language states that "although [these words] ${ }^{1}$ are traditionally analysed as adverbs, it is arguable that they do not in fact satisfy the traditional definition" (614). The authors propose a revision based on syntactic grounds, arguing that this class of words functions as a "goal complement with such verbs as come and $g o$ " (613). As adverbs are normally adjuncts and not complements, these words are more accurately classified as prepositions. They are unique among prepositions insofar as they cannot take noun phrases (NPs) as a complement, ${ }^{2}$ as shown in the diagram of the sample sentence "the girl came forward" below.



Figure 1: A syntactic tree showing the complementary nature of "forward."

\footnotetext{
${ }^{1}$ The words under discussion include several subclasses, such as deictic expressions (here, there), compass terms (north, south, east, west), and compounds (downhill, upstairs, indoors, underground), but I concern myself only with the subclass ending in -ward(s).

${ }^{2}$ Except for toward(s), which is my own inclusion.
} 
Authorities on the English language have not reached a consensus on the question of the distribution of the two forms, and are remarkably vague in their attempts to account for it. The Cambridge Grammar suggests that the major two factors at play are syntactic position and the split between British English (BrE) and American English (AmE): "Only the forms without $-s$ are used attributively ${ }^{3}$ (the homeward journey, a backward move). Elsewhere, $\mathrm{AmE}$ also normally uses the forms without $-s$, while $\mathrm{BrE}$ allows both forms, generally preferring the $-s$ variant (though forwards is more restricted in use than forward)" (Huddleston \& Pullum 2002). The last parenthetical note is not explained or developed further.

The Oxford English Dictionary takes a completely different approach to explaining the distinction between the $-\varnothing$ variant and the $-s$ variant. The entry on -ward(s) proposes several factors for differentiating between the two: "The two forms are so nearly synonymous...that the choice between them is mostly determined by some notion of euphony in the particular context; some persons, apparently, have a fixed preference for the one or the other form." Neither of these suggestions is linguistically satisfying: the idea of "euphony" needs to be defined by specific phonological, syntactic, or lexical features (e.g. a prevocalic environment, sentence-final position, or collocation), and the idea of individual preference being a major determiner suggests that the distinction is ultimately arbitrary and not systematic. The $O E D$ goes on to offer a third element to consider: "Sometimes, however, the difference in the form of the suffix corresponds to a difference in the shade of meaning conveyed, though it would not be possible to give any general rule that would be universally accepted." Again, there is evidence of hesitation in an authority's efforts to pinpoint the exact distinction between -ward and -wards. The examples given are that the $-s$ variant expresses a sense of manner as much as direction of movement, as in "to write backwards," and that this form also denotes more precise direction, so "to travel northwards" is more specific than "to travel northward."

A brief scan through online grammar resources reveals a similar lack of agreement as to when one variant should be preferred over another, whether it is a case of syntactic position, British versus American English, subtleties of meaning, one form gradually being supplanted by the other, or merely individual preference. To investigate this issue, then, my study must be diachronic, drawing from both sociolinguistic and historical methods of research.

Because prepositions ending in -ward have two possible and near-identical endings, $-\varnothing$ and $-s$, I hypothesize that (a) younger speakers will prefer the zero-ending form as it gradually replaces the other variant (see Figures 2 and 3 ), and (b) that the two forms will occur in distinctive environments (phonological, syntactic, or semantic) in issues of media publications.

\section{Methods}

\subsection{Subjects}

${ }^{3}$ When used attributively, their syntactic function becomes that of an adjective. 
To test this hypothesis, I took a population sample of native speakers of American-English divided into three age groups: Gen1 (18-35), Gen2 (3653 ), and Gen3 (54+). The divisions were based on increments of 18 , starting from 18 years old and working upward. To minimize the effects of variables that were less relevant to my research, I aimed to have 10-15 respondents in each age category. In the end, however, I had 15 subjects in both Gen 1 and Gen3, but only 2 in Gen2. As a result, I decided to focus my analysis exclusively on my youngest and oldest age groups, where I expected to have the greatest contrast anyway. By filtering my results for native speakers, I reduced interference from speakers' other native languages as well as level of English education. I specifically chose not to ask participants about their educational background for two reasons: that it might have led them to believe that the experiment is a test of their knowledge of the prescriptive rules of English grammar, which might have influenced their responses, and that the experiment was not testing such knowledge, but rather the natural tendencies of speech. Asking about the variable of education, then, was both immaterial and likely to affect data negatively.

I wanted my population sample to have a relatively balanced proportion of males to females, as gender was not the target of my study, but I ended up with an uneven ration of 9 males and 23 females, meaning that $72 \%$ of my respondents were women. Additionally, as dialect often plays a role in speech tendencies, I asked respondents to list the states and/or countries where they had resided for a year or more and give the amount of time they lived there. Results showed a wide geographical range, from Maine to Florida to California, with a concentration in New England and a few cases in which respondents had lived abroad in Canada, Chile, China, Germany, or Nicaragua, but never for longer than six years. To reassure my subjects of the confidentiality of their information, which they may consider sensitive, the top of the survey had a note assuring them that their information would not be disclosed for purposes beyond the scope of this project, and that it would not be traceable back to them.

My methods for recruiting participants were based on networking and media. I started by emailing a link to the survey to a listserve which I belong to, because the members represent an equal distribution of gender and age category and a fairly broad geographical area. I gathered ten responses from this technique, then turned to social media, posting the link on Facebook. While this helped me reach my objective in terms of number of respondents, the participants were predominately female and exclusively in the 18-35 and $54+$ age brackets.

\subsection{Materials}

This study has two aspects: a synchronic sociolinguistic aspect using apparent time and a diachronic historical linguistic aspect. For the sociolinguistic aspect, my primary form of data collection was an online survey, created with the website Qualtrics. Participants were given pairs of sentences that differed in only one word (the target word) and asked to choose the form that they preferred. Some of the sentences were written and others were recorded, as there may be some differences in respondents' 
perception of written versus spoken language. Since I may have my own biases that could be reflected in my utterance of the sentences, I asked someone else to read the sentences for me as I recorded them using a basic recording application for $\mathrm{iPad}$.

The survey itself started with a note that informed participants that this was not a test with right and wrong answers, but a study of actual language use, so they should select the answer that best resonated with their natural speech. Of the seventeen -ward words listed in the Cambridge Grammar of the English Language, I selected 12 - forward(s) and backward(s); inward(s) and $\operatorname{outward}(s) ;$ upward(s) and downward(s); onward(s); eastward(s), northward(s), and southward(s); homeward(s) and heavenward(s) - and added toward/s to give me a set of 13 .

I grouped the complementary sets of prepositions together into 6 sets (separated by semicolons in the list above) and came up with four pairs of sentences for each set, for a total of 24 pairs of sentences. I left 12 of these pairs in written form and recorded a volunteer saying the other 12 . To prevent subjects from becoming too concerned with consistency, I also added three "dummy" sentences involving the words amid(st), while/whilst, and among(st). Since there was a possibility that speakers' selections would be influenced by the syntactic and/or phonetic environment of the occurrence of the tokens, I deliberately varied these, putting the target word in both attributive and predicative positions where possible and positioning it to precede both consonants and vowels. On the final survey the sentences were presented in random order, with the dummy sentences mixed in. The first part of the survey (12 target sentences and 2 dummy sentences) required participants to choose between the two nearly identical variants, with the question, "Which of these two sentences do you prefer?" In the second part (12 target sentences and 1 dummy sentence), I asked respondents to give a rating of how natural they found each of the two sentences on a scale of one to five, illustrated with stars. The instruction was, "Rate these two sentences based on how natural they sound to you." This data was intended to reveal the strength of speakers' preferences, or possibly their ambivalence.

For the historical aspect of the study, I needed a searchable online corpus of a regular general interest publication over a long span of time. I chose The New Yorker from 1925 to the present, which meets these criteria. Although I recognize that this corpus, like many such publications, tends to be linguistically conservative and less accepting of colloquial forms, it will nevertheless provide a temporal cross-section of accepted written language.

\subsection{Procedure}

After I wrote the sample sentences and recorded half of them, I created a survey on Qualtrics and tested it on a couple of peers. Taking their feedback into account, I completed the survey and sent it out by email and social media to friends and family. When I had enough participants (30 was my goal), I closed the survey and analyzed the results.

For the historical study, I used Google Ngrams, an online search engine that charts the frequency of words in printed texts over a long span of time, in 
order to get a long-term perspective on the relative frequencies of the two coexisting forms. Next I ran a search in The New Yorker to narrow the focus of my corpus research. Finally, to resolve some issues from the sociolinguistic study, I pulled out examples of certain words in context and tried to make generalizations about the environments in which they appeared in one form rather than the other.

\subsection{Results}

\subsubsection{Sociolinguistic Data}

After acquiring the necessary information through Qualtrics and sorting the responses by age group, I tabulated the data into two Excel spreadsheets (Tables 1 and 2). In both tables, italics signify that the sentence was a recording, and " $\mathrm{R}$ " in the heading of Table 1 stands for "respondents." I highlighted percentages of 80 and higher in gold and percentages of below 20 in blue as an aide to making significant generalizations. Several individuals reported either in person or in the comments section at the end of the survey that they were unable to play the audio files, which accounts for the fact that there were fewer respondents for the italicized sentences than for the plain text ones. For this reason, I will focus my analysis on the general percentages rather than the more variable number of respondents.

Table 1: Respondents' Preferences of Sentence Variants by Age Group

\begin{tabular}{|c|c|c|c|c|c|c|c|c|}
\hline Sentences & Total R & -1 & Total \% $=$ & Gen1 R & $=$ & Gen1\% - & Gen3 R - & Gen3\% $=$ \\
\hline 1a. He was walking toward our house. & & 18 & $56 \%$ & & 5 & $33 \%$ & 12 & $80 \%$ \\
\hline 1b. He was walking towards our house. & & 14 & $44 \%$ & & 10 & $67 \%$ & 3 & $20 \%$ \\
\hline 2a. They left the boxes on the dock for onward shipment. & & 30 & $94 \%$ & & 14 & $93 \%$ & 14 & $93 \%$ \\
\hline $2 \mathrm{~b}$. They left the boxes on the dock for onwards shipment. & & 2 & $6 \%$ & & 1 & $7 \%$ & 1 & $7 \%$ \\
\hline 3a. Their southward journey took them to the mountains. & & 32 & $100 \%$ & & 15 & $100 \%$ & 15 & $100 \%$ \\
\hline 3b. Their southwards journey took them to the mountains. & & 0 & $0 \%$ & & 0 & $0 \%$ & 0 & $0 \%$ \\
\hline 4a. Kay took a step backward. & & 11 & $42 \%$ & & 6 & $43 \%$ & 4 & $40 \%$ \\
\hline 4b. Kay took a step backwards. & & 15 & $58 \%$ & & 8 & $57 \%$ & 6 & $60 \%$ \\
\hline 5a. The train continued onward through the tunnel. & & 30 & $94 \%$ & & 14 & $93 \%$ & 15 & $100 \%$ \\
\hline $5 \mathrm{~b}$. The train continued onwards through the tunnel. & & 2 & $6 \%$ & & 1 & $7 \%$ & 0 & $0 \%$ \\
\hline 6a. It was immediately clear to us that she had experienced an inward change & & 24 & $92 \%$ & & 13 & $100 \%$ & 9 & $82 \%$ \\
\hline 66. It was immediately clear to us that she had experienced an inwards chang & & 2 & $8 \%$ & & 0 & $0 \%$ & 2 & $18 \%$ \\
\hline 7a. The pulse expanded outward in circles from the point of impact. & & 17 & $53 \%$ & & 6 & $40 \%$ & 11 & $73 \%$ \\
\hline 7b. The pulse expanded outwards in circles from the point of impact. & & 15 & $47 \%$ & & 9 & $60 \%$ & 4 & $27 \%$ \\
\hline Sa. Helen turned toward the sea. & & 21 & $66 \%$ & & 5 & $33 \%$ & 15 & $100 \%$ \\
\hline Sb. Helen turned towards the sea. & & 11 & $34 \%$ & & 10 & $67 \%$ & 0 & $0 \%$ \\
\hline 9a Paul makes upward of $\$ 2000$ a month & & 8 & $32 \%$ & & 4 & $31 \%$ & 4 & $40 \%$ \\
\hline 9b. Paul makes upwards of $\$ 2000$ a month & & 18 & $72 \%$ & & 9 & $69 \%$ & 7 & $70 \%$ \\
\hline 10a. The tree's branches seem almost to grow downward. & & 21 & $66 \%$ & & 8 & $53 \%$ & 13 & $87 \%$ \\
\hline 10b. The tree's branches seem almost to grow downwards. & & 11 & $34 \%$ & & 7 & $47 \%$ & 2 & $13 \%$ \\
\hline 11a. He cast his eyes heavenward, seeking answers. & & 25 & $78 \%$ & & 9 & $60 \%$ & 15 & $100 \%$ \\
\hline 11b. He cast his eyes heavenwards, seeking answers. & & 7 & $22 \%$ & & 6 & $40 \%$ & 0 & $0 \%$ \\
\hline 12a. Children should be facing forward in their seats. & & 23 & $92 \%$ & & 11 & $85 \%$ & 10 & $100 \%$ \\
\hline 12b. Children should be facing forwards in their seats. & & 2 & $8 \%$ & & 2 & $15 \%$ & 0 & $0 \%$ \\
\hline
\end{tabular}

A few patterns emerge from studying the highlighted percentages in Table 1. Firstly, all subjects showed a marked or even exclusive preference for the $-\varnothing$ prepositions in the attributive position over the predicative, as in sample sentences 2, 3, and 6. This syntactic variable was suggested to be the most significant determiner by Huddleston and Pullum in The Cambridge Grammar of the English Language. 
The second meaningful pattern is that with the sole exceptions of 4,6 , and 9 (in which the difference was just 1\%), the younger participants of Gen1 had a higher rate of preference for the $-s$ form, meaning that they were more accepting of this variant. The greatest divergence between generations occurs with samples 1 (a 47\% difference) and 8 (a 67\% difference), which both involve the word toward(s). The younger generation seems to have a much stronger preference for the $-s$ variant of this particular word.

Finally, there are two cases in which both age groups prefer the $-s$ variant: sentences 4 and 9. These exceptions, Kay took a step backwards and Paul makes upwards of $\$ 2000$ a month, require corroborative data for an accurate analysis, and so for now they will simply be noted and set aside for future review.

Table 2: Respondents' Ratings of Sentence Variants by Age Group

\begin{tabular}{|l|r|r|r|}
\hline Sentences & Total Avg Rating & Gen1 Avg Rating & Gen3 Avg Rating \\
\hline 13a. Toward the end of the road, there was a playground. & 4.31 & 4.08 & 4.73 \\
\hline 13b. Towards the end of the road, there was a playground. & 3.79 & 4.07 & 3.31 \\
\hline 14a. An outward appearance is not always what it seems. & 4.74 & 4.6 & 4.86 \\
\hline 14b. An outwards appearance is not always what it seems. & 1.81 & 1.71 & 1.82 \\
\hline 15a. I took two steps forward. & 4.77 & 4.73 & 4.79 \\
\hline 15b. I took two steps forwards. & 1.88 & 2.07 & 1.7 \\
\hline 16a. We started getting tired toward the end of the second act. & 3.94 & 3.67 & 4.07 \\
\hline 16b. We started getting tired towards the end of the second act. & 3.93 & 4.21 & 3.42 \\
\hline 17a. Our onward journey would take us over the mountains. & 4.55 & 4.43 & 4.77 \\
\hline 17b. Our onwards journey would take us over the mountains. & 1.79 & 1.46 & 2.22 \\
18a. Jim gave her a backward glance. & 3.48 & 3.07 & 3.71 \\
\hline 18b. Jim gave her a backwards glance. & 3.9 & 4 & 3.92 \\
\hline 19a. Before you can move onward, I need to see your papers. & 4.53 & 4.4 & 4.6 \\
\hline 19b. Before you can move onwards, I need to see your papers. & 1.93 & 1.87 & 1.83 \\
\hline 20a. When I look at the lake, my thoughts turn inward. & 4.61 & 4.57 & 4.67 \\
\hline 20b. When I look at the lake, my thoughts turn inwards. & 2.48 & 2.46 & 2.25 \\
\hline 21a. The wind blew the boat eastward into the current. & 4.59 & 4.57 & 4.62 \\
\hline 21b. The wind blew the boat eastwards into the current. & 2 & 1.77 & 2 \\
\hline 22a. Frustrated, Lou flung the ball upward. & 4.16 & 4 & 4.43 \\
\hline 22b. Frustrated, Lou flung the ball upwards. & 3.22 & 3.38 & 2.83 \\
\hline 23a. The downward pull of gravity would not be defied. & 2.81 & 4.67 & 4.93 \\
\hline 23b. The downwards pull of gravity would not be defied. & 4.14 & 2.63 & 3.91 \\
\hline 24a. After three long weeks, I was finally headed homeward. & & 2.46 & 2.67 \\
\hline 24b. After three long weeks, I was finally headed homewards. & & 4.42 \\
\hline
\end{tabular}

In Table 2, which represents the data collected in the second half of the survey, I recorded the average ratings (on a scale of 1-5) of the two sentence variants by respondents from the two age groups. Averages of 4 or greater are highlighted in gold, and averages of 2 or lower are highlighted in blue. One fact that I overlooked while designing this part of the survey is that when respondents wished to give a rating of 0 by not selecting any stars, this response was recorded as an unanswered question. As a result, ratings do not fall below 1 and so I have raised the threshold to 2 to compensate for this slightly skewed data.

The first trend that is immediately apparent in this table is that all subjects, regardless of age group, tend to give the $-\varnothing$ form a higher rating. However, there are several exceptions to this tendency which should be examined. In sentences 13 and 16, respondents from Gen 1 gave the $-s$ form a rating higher than or nearly equal to their rating of the $-\varnothing$ form, while Gen 3 gave average ratings of 3.31 and 3.42 respectively, which represent their second and third 
highest ratings of $-s$ variants in the data sample. Both 13 and 16 contain the target preposition toward(s). As in Table 1, then, we see that towards is one of the most strongly favored -wards prepositions, and that the younger generation prefers it more than the older generation.

The other special exception to the dominance of the $-\varnothing$ form is the case of backwards in sentence 18: Jim gave her a backward(s) glance. Both age groups gave a higher rating for the $-s$ form, even though it was in the attributive position. It should be noted that $14 \mathrm{~b}, 17 \mathrm{~b}$, and $23 \mathrm{~b}$ each contained an $-\mathrm{s}$ variant in the attributive position, and these three sentences received some of the lowest ratings in the dataset from both age groups. Also, as we saw in Table 1, being in this syntactic position significantly contributed to respondents' dispreference for the $-s$ variant. However, in Table 1 we also have the exceptional case of Kay took a step backwards, which was generally preferred over the sentence with the $-\varnothing$ variant. The common feature here is backwards, which seems to be favored over backward, regardless of its syntactic position.

\subsubsection{Corpus Data}

To gain a broad overview of the frequency of occurrences of tokens over time, I used Google Ngrams, which finds specific words in the vast corpus of google books over 200 years (1800-2000). Because of the substantial difference in the frequency of toward(s) and forward(s) with respect to the other words, I plotted these words on a separate graph (Figure 3 ) along with backward(s), to provide a link between Figures 2 and 3 and to give some perspective. I omitted the five words with the lowest overall frequency (eastward(s), northward(s), southward(s), heavenward(s) and homeward(s)) to avoid cluttering the graph excessively.

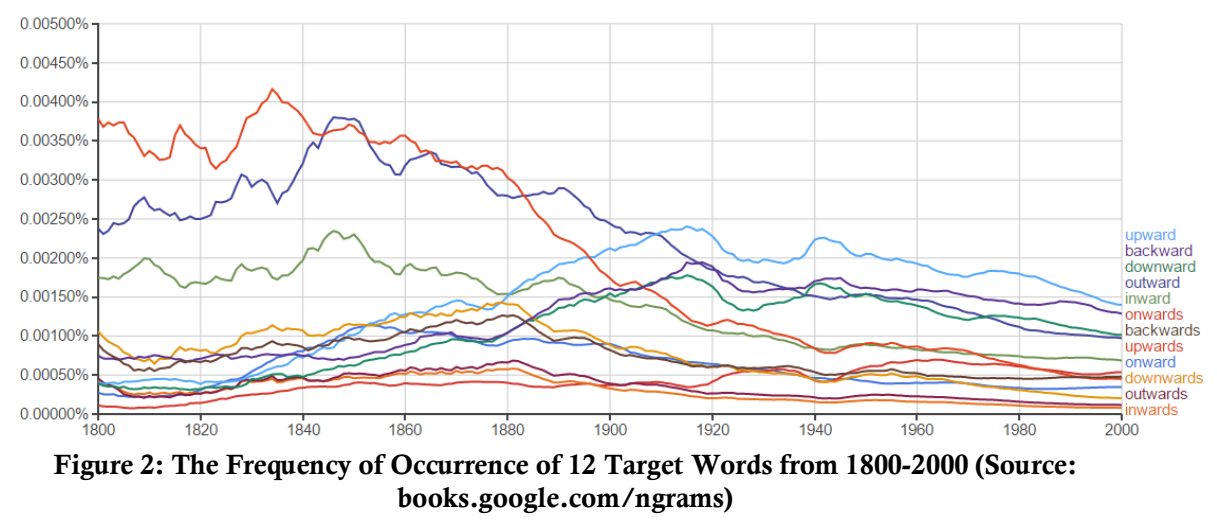




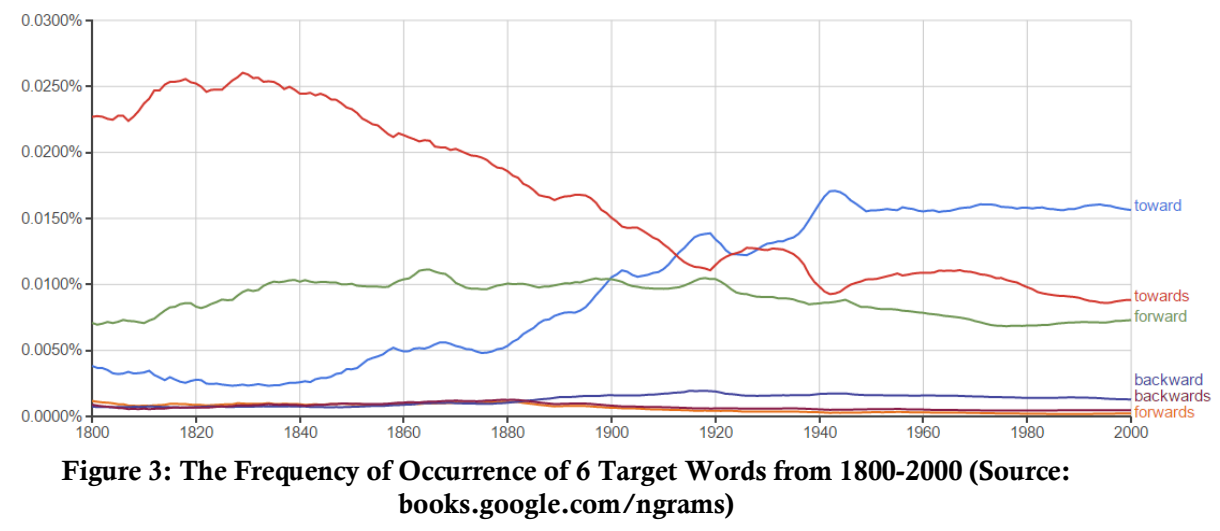

As these graphs are only intended to give a general background, I will not analyze the course of each target word in depth. I do, however, want to observe a few interesting trends. Surprisingly, the pairs of words follow very different patterns of development: upward and toward both take over from their $-s$ forms in a crossover that occurred around 1910, while forward maintains a fairly consistent position over forwards, and onward and onwards seem to be close to merging. This discrepancy of development patterns perhaps suggests that these words are not regarded as a coherent class of words and should be treated as distinct from one another. The few generalizations that can be made are that, with the exception of onward(s), the $-\varnothing$ variant is always the dominant form by the year 2000 , and that there is an overall movement toward convergence. This convergence suggests that both forms are equally accepted, which means that perhaps they are taking on specialized meanings so that they will continue to exist, but with differences in use or implication.

For a more specific historical linguistic approach to this matter, I have chosen to analyze a corpus consisting of all issues of The New Yorker, a magazine that contains writing samples in many different styles (poetry, fiction, essays, criticism, etc.) published at regular intervals over the past 90 years. Figure 4 provides a preliminary look at the relative frequency with which the two variants of the target words occur in this magazine. The data is inaccurate because the magazine's search function caps at 999 tokens, but there are still some conclusions to be drawn from the graph. 




Figure 4: Comparative bar graph of the two variants of the target words in The New Yorker, 1925-2015

It is clear from Figure 3 that the $-\varnothing$ ending dominates the pages of The New Yorker. The relative frequencies of toward and towards cannot be determined from the information given since both appear more than 1000 times in the corpus, and the numbers for forward(s) are imprecise because some of the tokens refer to the homonymous position in soccer. Other numbers are imprecise because they represent a repeated occurrence of the same sentence; homewards, for example, is listed as occurring 5 times, but 4 of those times are in the same advertisement appearing in 4 different issues. This type of corpus search, then, presents many challenges to making accurate generalizations. What can safely be abstracted from it is the predominance of the zero-ending form in the magazine. Because this variant is so much more common, I will consider it the default (less marked in terms of frequency of use) form and limit my study of context to the more marked $-s$ variants.

Corpus research can also provide us with a view of varying contexts in which tokens occur and resolve some issues that were raised in the sociolinguistic study. The target words that showed the most significant variation in the data gathered by the survey were backwards, which was slightly preferred over backward even in attributive position, upwards, which was preferred in one sample sentence but not in another, and towards, which was regarded differently by subjects from different generations.

Below I list some extracts of the target words in chronological order with their immediate context and date of issue:

\section{Backwards}

a) The guard gets people who skate backwards. [Dec 24, 1927 p15]

b) If there is a wind blowing, the machine will even drift backwards. [Nov 1, $1930 \mathrm{p} 10$ ] 
c) ...the possibility that the machine might be made to run backwards. They like, for instance, to run home-movie projectors backwards in order to see divers appear. [May 20, 1933 p22]

d) He imagined that, if only the oboe sonata were played backwards, they would leap backwards from the telephone pole and be on their way home again. [Mar 11, 1967 p51]

e) The stamp was printed backwards. [Oct 5, 1968 p115]

f) Philosophers say, that life must be understood backwards. [Sept

2, 1972 p26]

g) Three months later, I was dunking everything, every way - with two hands, backwards, backwards with two hands. [...] You know, you jump up backwards. [Mar 22, 1993 p80]

h) Is it possible to go backwards and forwards at the same time?

[Mar 16, 1998 pCV2]

i) Straightforward. As opposed to backwards and crooked. [Aug 31, 2009 pCV2]

2. Upwards

j) Just off Washington Square[,] convenient[,] homelike[,] reasonable[:] 1 room...\$65 upwards[,] 2 rooms...\$85 upwards[,] 3 rooms...\$115 upwards [Sept 28, 1929 p90]

k) Imagine paying from $\$ 1900, \$ 3050$ or $\$ 2900$ upwards for 6 , 9, or

10 rooms in a building like 520. [May 27, 1933 p29]

1) Solitaire Rings mounted with perfect diamonds, $\$ 500$ upwards. [January 25, $1947 \mathrm{p} 49$ ]

m) At 334 bookstores, New Yorkers spend upwards of $\$ 18,000,000$ a year. [Dec 3, $1960 \mathrm{p} 86]$

$\mathrm{m})$ Today's automobile driver guides a ton or more of complex machinery at upwards of 80 feet per second through an unpredictable world. [Apr 4, 1983 p65]

\section{Towards}

n) "What'sa matter, Billie?" he asked, quietly. "Your feelings towards me changed?" "My feelings towards you?" [Sept 8, 1934 p25]

o) Together they lifted the gnome and threw him out towards the center of the pool. [Aug 18, 1951 p35]

p) She pushed Harry away and began to walk down the lane towards her home. [May 11, $1957 \mathrm{p} 42$ ]

q) He represents the ideal towards which historians strive - or rather (to avoid generalizing in my turn) towards which I, as a historian, strive. [Dec 8, 1962 p101]

r) ...fond observer of the classic American ambivalence towards heterosexual psychoses [Feb 4, 1967 p122]

s) And straight away I am looking towards the next thing. [Sept 3 , 1990 p37]

t) Until now, everyone has best worked in his place in the new Germany if, so to speak, he works towards the Fuhrer. [Mar 8, 1999 p90]

u) Use points towards both hotel rooms and flights. [Jan 8, 2007 p3] 
v) And that will go far towards powering lives for decades to come.

[Dec 22, 2014 p6]

It is not always easy or straightforward to reach conclusions about the use of these words, but some generalizations can be hazarded. As far as backwards is concerned, it may be the case that, as the OED proposed, there is a distinction in meaning between the two variants. Examples a, c, the first token in $\mathrm{d}, \mathrm{e}, \mathrm{f}, \mathrm{g}$, and possibly $\mathrm{i}$ all seem to denote a manner of action rather than a direction, but $\mathrm{b}$, the second token in $\mathrm{d}$, and $\mathrm{h}$ all clearly indicate $\mathrm{a}$ direction of movement. This semantic distinction, then, may not be fully defined.

Upwards is a simpler matter to resolve. All of the uses cited, even the earliest ones, are in the context of quantities and denote an unknown upper limit. This is a specialized use of upwards and explains why survey respondents favored 9b: Paul makes upwards of $\$ 2000$ a month, but were more ambivalent about 22b: Frustrated, Lou flung the ball upwards.

Towards, like backwards, seems to have a special semantic value. With the exceptions of $\mathrm{o}, \mathrm{p}$, and arguably $\mathrm{s}$, in each of these contexts towards has an abstract rather than a literal meaning. All of my examples with this word in the survey described literal direction and were nevertheless largely accepted, especially by the younger generation, but that does not negate the fact that it has a specialized use and meaning.

\section{Discussion}

I shall now attempt to synthesize the results from my sociolinguistic and historical methods of data collection and see if they upheld my hypothesis. First of all, I proposed that younger speakers would prefer the zero-ending form. This supposition was based on a trend I had observed in the ngrams of Figures 1 and 2, that toward had started to take over from towards. However, based on my sociolinguistic data, this hypothesis is false and in fact the opposite is true: younger speakers are actually more favorable to the sending forms.

I cannot fully account for this phenomenon with the data at hand. Perhaps my Gen3 participants were more conscious of the "correctness" of the unmarked default form; as one Gen3 wrote in the final comments section of the survey, "The extra $\mathrm{S}$ is commonly added in speech by the blessedly unconcerned." The respondent here expresses his/her belief that the zeroending variant is always the correct one, and makes a further qualification of "in speech," suggesting that this is a colloquialism that is less acceptable in standardized written language. Another possible explanation for the preferences of the younger generation is that there is another paradigm shift in the works, that $-s$ variants are regaining ground as they find specialized uses and meanings. Certainly it seems unlikely that one form will totally supplant the other in the near future.

The second part of my hypothesis, that the two forms would occur in distinctive environments (phonological, syntactic, or semantic) in issues of media publications, proved true to a certain extent. There was no evidence that phonological environment played a role either in subjects' selection of 
sentences or in the examples drawn from The New Yorker. Syntactic position did have an influence, as Huddleston and Pullum suggested, because - $\varnothing$ variants were preferred in attributive position. Semantic distinctions, the $O E D$ 's "shade[s] of meaning," also helped determine which word was favored in which context, as in the case of backward(s), where there is a distinction between manner and direction; upwards, which occurs in contexts of vertically unbounded quantities; and towards, which is preferred for indication of abstract or metaphorical direction.

In short, neither syntax nor semantics alone can determine which variant will be preferred, nor does it seem from this limited data sample that age is an adequate deciding factor. All of these elements come together in a sociolinguistic matrix that subconsciously shapes an individual's preference for one form over another. As one Gen1 respondent noted in the comments: "It's interesting how some [of the sentences] sounded okay while others didn't. I was never set on one or the other [of the variants]."

Yet another element to be considered is the difference between formal and colloquial language. The proportions of -ward to -wards variants in The New Yorker, as graphed in Figure 4, do not seem consistent with the preferences of the survey subjects, who were much more willing to accept the $-s$ forms. This indicates that the zero-ending variant may be regarded as the more "correct" version in formal writing, while the s-ending is reserved for casual speech, as the Gen3 respondent suggested. Register, then, may be another factor to take into account.

\section{Conclusion}

Ultimately it may not be productive to consider all the -ward(s) prepositions as belonging to a single class just due to their shared bound suffix. The words have undergone different developmental process over time, and have started to differentiate themselves on syntactic and semantic grounds that are unique for each pair of words.

This particular study had its faults, among them skewed gender and age demographics, inaccuracies due to problems with the audio not working and the 0 -rating in the second half being interpreted as no response, the difficulty of narrowing a vast amount of information into a feasible corpus study, and the attempts to make generalizations based on items that do not necessarily constitute a coherent, homogeneous class. However, I believe that this study is useful for recognizing that there are a number of factors, historical and social as well as contextual, that contribute to an individual's decision when choosing between two nearly identical forms, and that such forms are unlikely to coexist without diverging in some aspect of their syntactic distribution or semantic denotation. 